OPEN ACCESS

International Journal of

Environmental Research and

Public Health

ISSN 1660-4601

www.mdpi.com/journal/ijerph

Article

\title{
Modeling the Effects of Indoor Passive Smoking at Home, Work, or Other Households on Adult Cardiovascular and Mental Health: The Scottish Health Survey, 2008-2011
}

\author{
Ivy Shiue ${ }^{1,2}$
}

1 School of the Built Environment, Heriot-Watt University, Riccarton, EH14 4AS, Edinburgh, Scotland EH14 4AS, UK; E-Mail: I.shiue@hw.ac.uk; Tel.: +44-131-451-4655;

Fax: +44-131-451-3161

2 Owens Institute for Behavioral Research, University of Georgia, 215 S Jackson St. Athens, GA 30602, USA

Received: 4 February 2014; in revised form: 27 February 2014 / Accepted: 4 March 2014 /

Published: 13 March 2014

\begin{abstract}
Passive smoking has contributed increased risks of cardiovascular disease, mental health, and mortality, but the cumulative effects from work or other households were less studied. Therefore, it was aimed to model the effects of indoor passive smoking from own home, work, and other households in a country-wide, population-based setting. Data in the Scottish Health Survey between 2008 and 2011 after the law banning smoking in public places were analyzed. Information including demographics, lifestyle factors, and self-reported cardiovascular disease and mental health was obtained by household interview. Analyses included chi-square test and survey-weighted logistic regression modeling. After full adjustment, it was observed that being exposed to indoor passive smoking, in particular in more than two places of exposure, was significantly associated with risks of stroke, angina, heart attack, abnormal heart rhythms, and GHQ $\geq 12$. The significance remained for angina, GHQ $\geq 12$ and probably heart attack in never smokers. The cumulative risks also impacted on sleep problems, self-recognition, making decisions, self-confidence, under strain constantly, depressed, happiness and self-worth. The significance remained for sleep problems, self-confidence, under strain constantly, depressed, and happiness in never smokers. Elimination of indoor passive smoking from different sources should still be a focus in future public health programs.
\end{abstract}

Keywords: housing; public opinion; policy; public health; homeless; social determinants 


\section{Introduction}

It has been known that passive smoking has contributed increased risks of cardiovascular disease (CVD), mental health, and mortality including in Scotland (from data between 1998 and 2003) [1]. Since the 1980s until recent years [2] there have been several scientific review articles illustrating the worldwide hazard effect of passive smoking on occupants across age groups with various health concerns. The law banning smoking in public places in Scotland has been approved and implemented after scientific evidence was provided [3]. The considerable benefits achieved included air quality improvement, avoidance of adverse health outcomes and broader policy empowerment in Scotland, while the importance of learning from other administrations, and political and professional leadership were also noted until 2008. Further, restriction of passive smoking in households has proved to be protective for children in Scotland [4]. However, the risk effects from different sources other than own home or the cumulative risk effects by counting number of indoor places were less studied. Following the context, therefore, the aim of this work was to model the cumulative risk effects of indoor passive smoking from own home, work, and other households in a country-wide, population-based setting in adults using data from after 2008.

\section{Experimental Section}

\subsection{Study Sample and Variables}

As described elsewhere in detail [5], the Scottish Health Survey [6] has been a country-wide, population-based, multi-year study. It provides a detailed picture of the health of the Scottish population in private households and is designed to make a major contribution to the monitoring of health in Scotland. It is essential for the Scottish Government's forward planning, for identifying gaps in the provision of health services and for identifying which groups are at particular risk of future ill-health. More survey design details, including sample size estimation, can be found online [7]. In the present study, the most recent four years of available data from 2008 to 2011, consisting of four cycles of study cohorts $(\mathrm{N}=36,922)$, after the law banning passive smoking in households were combined for examination. Information including demographics, experiences on being exposed to indoor passive smoking and self-reported health conditions were obtained by household interview. The age range was from 0 to 103 , but the adult participants included for the current analysis were those aged between 18 and 103 since information on $\mathrm{CV}$ and mental health were obtained in adults only. Study exposures were "whether exposed to passive smoking at home, at work, or at other people's home (are you regularly exposed to other people's tobacco smoke in any of these places?)" while study outcomes were self-reported CVD, including stroke, angina, heart attack, heart murmur, abnormal heart rhythm, and other heart problems and mental health status by General Health Questionnaire (GHQ). GHQ-12 has been utilized to assess subject's mental health and psychological distress status [8]. A cut-off of 12 was used to screen people with psychological distress (GHQ score $\geq 12$ ). 


\subsection{Statistical Analysis}

Statistical analyses included chi-square test and logistic regression modeling and models were weighted for survey design. Covariates including age, sex, ethnicity, body mass index (BMI), smoking status, high blood pressure, education level, regular exercise, and alcohol intake were adjusted in the modeling. In the subsequent analysis, never smokers were examined separately. Effects were reported in odds ratios (OR) from general logistic regression models or relative risk ratios (RRR) from multi-level logistic regression models depending on the study outcomes being binary (mainly self-reported CVDs) or ordinal (mainly self-reported mental health status) together with 95\% confidence intervals, with $p<0.05$ considered statistically significant. The statistical software STATA version 13.0 (STATA, College Station, Texas, USA) was used to perform all the analyses. Since this study is only a secondary data analysis based on extracting data from the UK Data Archive website, no further ethics approval is required. The survey was reviewed by an independent Research Ethics Committee and given a favourable opinion by the Cardiff Research Ethics Committee before collecting the primary data [9].

\section{Results and Discussion}

\subsection{Main Results}

Of all included adult participants $(\mathrm{N}=27,998), 56.5 \%$ were female, 9,087 (32.5\%) had education less than high school, while only 6,997 (25.0\%) had a normal BMI (18.5-25). Other characteristics were shown in Table 1. In Table 2, the relationships of indoor passive smoking and several self-reported CVDs are shown. After full adjustment (see footnote in the Table 2), it was observed that being exposed to passive smoking in more than two places could largely increase risks of total CVD (OR 1.35, 95\%CI 1.11-1.65, $p=0.003$ ), stroke (OR 1.88, 95\%CI 1.28-2.76, $p=0.001$ ), angina (OR 1.50, 95\%CI 1.06-2.11, $p=0.022$ ), heart attack (OR 1.87, 95\%CI 1.29-2.71, $p=0.001$ ), abnormal heart rhythms (OR 1.36, 95\%CI 1.07-1.74, $p=0.013$ ), and GHQ $\geq 12$ (OR 1.45, 95\%CI 1.26-1.67, $p<0.001)$, but not heart murmur, diabetes, or other heart problems.

Table 1. Characteristics of the whole study cohort and the included adult cohort for analysis.

\begin{tabular}{cc}
\hline $\mathbf{( N}=\mathbf{3 6 , 9 2 2})$ & $\mathbf{N}(\mathbf{\%})$ or Mean $(\mathbf{S D})$ \\
\hline Age (range: 0-103) & $41.1(24.3 \%)$ \\
$0-29$ & $12,447(33.7 \%)$ \\
$30-59$ & $14,430(39.1 \%)$ \\
$60-79$ & $8,410(22.8 \%)$ \\
$80-99$ & $1,632(4.4 \%)$ \\
$100-103$ & $3(0.01 \%)$ \\
Sex & \\
Male & $16,693(45.2 \%)$ \\
Female & $20,229(54.8 \%)$ \\
\hline
\end{tabular}


Table 1. Cont.

\begin{tabular}{|c|c|}
\hline$(\mathrm{N}=36,922)$ & N (\%) or Mean (SD) \\
\hline \multicolumn{2}{|c|}{ Adult Cohort (aged 18-103; $n=27,998)$} \\
\hline \multicolumn{2}{|l|}{ Sex } \\
\hline Male & $12,170(43.5 \%)$ \\
\hline Female & $15,828(56.5 \%)$ \\
\hline \multicolumn{2}{|l|}{ Education } \\
\hline$<$ high school & $9,087(32.5 \%)$ \\
\hline$\geq$ high school & $18,848(67.5 \%)$ \\
\hline \multicolumn{2}{|l|}{ Body mass index } \\
\hline$<18.5$ & $283(1.0 \%)$ \\
\hline $18.5-25$ & $6,997(25.0 \%)$ \\
\hline $25-30$ & $8,930(31.9 \%)$ \\
\hline $30-40$ & $6,392(22.8 \%)$ \\
\hline 40 and over & $648(2.3 \%)$ \\
\hline Missing & $4,748(17.0 \%)$ \\
\hline \multicolumn{2}{|l|}{ Smoking nowadays } \\
\hline Current & $6,837(24.2 \%)$ \\
\hline Past & $10,252(36.6 \%)$ \\
\hline Never & $10,912(39.0 \%)$ \\
\hline \multicolumn{2}{|l|}{ Secondhand smoking } \\
\hline Exposed to 0 place & $21,268(76.0 \%)$ \\
\hline Exposed to 1 place & $5,388(19.2 \%)$ \\
\hline Exposed to $\geq 2$ places & $1,342(4.8 \%)$ \\
\hline
\end{tabular}

Table 2. Associations of indoor passive smoking and adult CVD.

\begin{tabular}{|c|c|c|c|c|c|c|}
\hline & \multirow{2}{*}{$\begin{array}{c}\text { Yes } \\
n(\%)\end{array}$} & \multirow{2}{*}{$\begin{array}{c}\text { No } \\
n(\%)\end{array}$} & \multicolumn{2}{|c|}{ Being Exposed in 1 Place } & \multicolumn{2}{|c|}{ Being exposed in $\geq 2$ Places } \\
\hline & & & OR $(95 \% C I)$ & $p$ value & OR $(95 \% C I)$ & $p$ value \\
\hline Total CVD & $10,456(37.4 \%)$ & $17,501(62.6 \%)$ & $1.25(1.13-1.37)$ & $<0.001$ & $1.35(1.11-1.65)$ & 0.003 \\
\hline Stroke & $943(3.4 \%)$ & $27,041(96.6 \%)$ & $1.53(1.27-1.86)$ & $<0.001$ & $1.88(1.28-2.76)$ & 0.001 \\
\hline Angina & $1,698(6.1 \%)$ & $26,272(93.7 \%)$ & $1.42(1.22-1.65)$ & $<0.001$ & $1.50(1.06-2.11)$ & 0.022 \\
\hline Heart attack & $1,177(4.2 \%)$ & $26,807(95.8 \%)$ & $1.36(1.14-1.61)$ & 0.001 & $1.87(1.29-2.71)$ & 0.001 \\
\hline Heart murmur & $1,161(4.2 \%)$ & $26,822(95.8 \%)$ & $1.19(1.00-1.42)$ & 0.051 & $1.17(0.84-1.64)$ & 0.360 \\
\hline Abnormal heart rhythms & $2,140(7.7 \%)$ & $25,835(92.3 \%)$ & $1.11(0.97-1.26)$ & 0.130 & $1.36(1.07-1.74)$ & 0.013 \\
\hline Other heart problems & $833(3.0 \%)$ & $27,159(97.0 \%)$ & $1.23(1.00-1.51)$ & 0.051 & $0.88(0.55-1.39)$ & 0.581 \\
\hline Diabetes & $1,809(6.5 \%)$ & $26,181(93.5 \%)$ & $1.11(0.96-1.28)$ & 0.145 & $1.16(0.85-1.58)$ & 0.354 \\
\hline GHQ-12 scores $\geq 12$ & $9,009(35.2 \%)$ & $16,553(64.8 \%)$ & $1.28(1.18-1.38)$ & $<0.001$ & $1.45(1.26-1.67)$ & $<0.001$ \\
\hline
\end{tabular}

Note: Adjusted for age, sex, ethnicity, body mass index, smoking status, high blood pressure, education level, alcohol intake, and exercise, and survey weighting.

To further explore events within mental health assessment scale (GHQ-12), it was additionally observed that the indoor passive smoking risk effect by accounting the number of exposing places could also impact on sleep problems (more than usual: OR 2.25, 95\%CI 1.63-3.11, $p<0.001$ ), self- recognition (less than usual: OR 1.83, 95\%CI 1.18-2.85, $p=0.007$ ), making decisions (less than usual: OR 2.09, 95\%CI 1.12-3.90, $p=0.020$ ), self-confidence (less than usual OR 2.27, 
95\%CI 1.64-3.12, $p<0.001$ ), under strain constantly (more than usual: OR 1.70, 95\%CI 1.22-2.37, $p=0.002$ ), depressed (more than usual: OR 2.44, 95\%CI 1.76-3.39, $p<0.001$ ), happiness (much less than usual: OR, 2.15, 95\%CI 1.40-3.30, $p<0.001$ ) and self-worth (much less than usual: OR, 1.74, 95\%CI 1.18-2.58, $p=0.006$ ), in particular when being exposed in more than two places (for details see Table 3).

Table 3. Associations of indoor passive smoking on adult mental health by GHQ-12 items.

\begin{tabular}{|c|c|c|c|c|c|}
\hline \multirow{2}{*}{ GHQ-12 Items } & \multirow{2}{*}{$\begin{array}{c}\text { Yes } \\
n(\%)\end{array}$} & \multicolumn{2}{|c|}{ Being Exposed in 1 Place } & \multicolumn{2}{|c|}{ Being Exposed $\geq 2$ Places } \\
\hline & & RRR $(95 \% C I)$ & $p$ value & RRR $(95 \% C I)$ & $p$ value \\
\hline \multicolumn{6}{|l|}{ Able to concentrate } \\
\hline better than usual & $779(2.8 \%)$ & reference & $\mathrm{n} / \mathrm{a}$ & reference & $\mathrm{n} / \mathrm{a}$ \\
\hline same as usual & $24,033(86.1 \%)$ & $1.13(0.89-1.43)$ & 0.319 & $0.97(0.62-1.54)$ & 0.991 \\
\hline less than usual & $2,658(9.5 \%)$ & $1.43(1.11-1.85)$ & 0.006 & $1.59(0.97-2.61)$ & 0.068 \\
\hline much less than usual & $450(1.6 \%)$ & $1.94(1.38-2.72)$ & $<0.001$ & $1.50(0.81-2.76)$ & 0.197 \\
\hline \multicolumn{6}{|l|}{ Lost sleep over worry } \\
\hline not at all & $10,249(36.7 \%)$ & reference & $\mathrm{n} / \mathrm{a}$ & reference & $\mathrm{n} / \mathrm{a}$ \\
\hline no more than usual & $13,310(47.6 \%)$ & $1.15(1.06-1.25)$ & 0.001 & $0.93(0.80-1.08)$ & 0.341 \\
\hline rather more than usual & $3,452(12.4 \%)$ & $1.32(1.17-1.48)$ & $<0.001$ & $1.10(0.87-1.38)$ & 0.418 \\
\hline much more than usual & $927(3.3 \%)$ & $1.85(1.53-2.23)$ & $<0.001$ & $2.25(1.63-3.11)$ & $<0.001$ \\
\hline \multicolumn{6}{|c|}{ Felt playing useful part in things } \\
\hline more than usual & $2,098(7.5 \%)$ & reference & $\mathrm{n} / \mathrm{a}$ & reference & $\mathrm{n} / \mathrm{a}$ \\
\hline same as usual & $22,872(82.1 \%)$ & $1.05(0.92-1.21)$ & 0.456 & $1.22(0.93-1.59)$ & 0.148 \\
\hline less than usual & $2,255(8.1 \%)$ & $1.40(1.17-1.67)$ & $<0.001$ & $2.27(1.63-3.17)$ & $<0.001$ \\
\hline much less than usual & $645(2.3 \%)$ & $1.65(1.27-2.14)$ & $<0.001$ & $1.83(1.18-2.85)$ & 0.007 \\
\hline \multicolumn{6}{|c|}{ Felt capable of making decisions } \\
\hline more than usual & $1,722(6.2 \%)$ & reference & $\mathrm{n} / \mathrm{a}$ & reference & $\mathrm{n} / \mathrm{a}$ \\
\hline same as usual & $24,202(86.6 \%)$ & $0.95(0.81-1.10)$ & 0.471 & $1.04(0.79-1.36)$ & 0.806 \\
\hline less than usual & $1,684(6.0 \%)$ & $1.21(0.98-1.48)$ & 0.073 & $1.61(1.11-2.33)$ & 0.012 \\
\hline much less than usual & $328(1.2 \%)$ & $1.79(1.30-2.45)$ & $<0.001$ & $2.09(1.12-3.90)$ & 0.020 \\
\hline \multicolumn{6}{|l|}{ Felt constantly under strain } \\
\hline not at all & $7,544(27.0 \%)$ & reference & $\mathrm{n} / \mathrm{a}$ & reference & $\mathrm{n} / \mathrm{a}$ \\
\hline no more than usual & $15,244(54.6 \%)$ & $1.05(0.96-1.15)$ & 0.293 & $0.93(0.78-1.12)$ & 0.449 \\
\hline rather more than usual & $4,182(15.0 \%)$ & $1.21(1.07-1.36)$ & 0.002 & $1.07(0.86-1.35)$ & 0.544 \\
\hline much more than usual & $958(3.4 \%)$ & $1.50(1.24-1.82)$ & $<0.001$ & $1.70(1.22-2.37)$ & 0.002 \\
\hline \multicolumn{6}{|c|}{ Felt couldn't overcome difficulties } \\
\hline not at all & $10,817(38.7 \%)$ & reference & $\mathrm{n} / \mathrm{a}$ & reference & $\mathrm{n} / \mathrm{a}$ \\
\hline no more than usual & $14,235(51.0 \%)$ & $1.20(1.11-1.31)$ & $<0.001$ & $1.00(0.87-1.15)$ & 0.966 \\
\hline rather more than usual & $2,257(8.1 \%)$ & $1.42(1.24-1.63)$ & $<0.001$ & $1.48(1.16-1.90)$ & 0.002 \\
\hline much more than usual & $611(2.2 \%)$ & $1.98(1.59-2.49)$ & $<0.001$ & $1.41(0.96-2.07)$ & 0.080 \\
\hline \multicolumn{6}{|c|}{ Able to enjoy day-to-day activities } \\
\hline more than usual & $1,403(5.0 \%)$ & reference & $\mathrm{n} / \mathrm{a}$ & reference & $\mathrm{n} / \mathrm{a}$ \\
\hline same as usual & $22,192(79.4 \%)$ & $1.03(0.85-1.24)$ & 0.769 & $1.15(0.84-1.58)$ & 0.384 \\
\hline less than usual & $3,478(12.5 \%)$ & $1.32(1.07-1.62)$ & 0.010 & $1.68(1.216-2.41)$ & 0.005 \\
\hline much less than usual & $863(3.1 \%)$ & $1.55(1.19-2.02)$ & 0.001 & $1.59(1.00-2.53)$ & 0.051 \\
\hline
\end{tabular}


Table 3. Cont.

\begin{tabular}{|c|c|c|c|c|c|}
\hline \multirow{2}{*}{ GHQ-12 Items } & \multirow{2}{*}{$\begin{array}{c}\text { Yes } \\
n(\%)\end{array}$} & \multicolumn{2}{|c|}{ Being Exposed in 1 Place } & \multicolumn{2}{|c|}{ Being Exposed $\geq 2$ Places } \\
\hline & & RRR (95\%CI) & $p$ value & RRR $(95 \% C I)$ & $p$ value \\
\hline \multicolumn{6}{|l|}{ Been able to face problems } \\
\hline more than usual & $1,212(4.3 \%)$ & reference & $\mathrm{n} / \mathrm{a}$ & reference & $\mathrm{n} / \mathrm{a}$ \\
\hline same as usual & $24,314(87.1 \%)$ & $0.96(0.79-1.17)$ & 0.683 & $0.71(0.52-0.98)$ & 0.037 \\
\hline less than usual & $1,975(7.1 \%)$ & $1.36(1.09-1.70)$ & 0.007 & $1.17(0.80-1.72)$ & 0.414 \\
\hline much less than usual & $420(1.5 \%)$ & $1.44(1.03-2.01)$ & 0.033 & $1.05(0.58-1.88)$ & 0.880 \\
\hline \multicolumn{6}{|c|}{ Been feeling unhappy and depressed } \\
\hline not at all & $11,937(42.7 \%)$ & reference & $\mathrm{n} / \mathrm{a}$ & reference & $\mathrm{n} / \mathrm{a}$ \\
\hline no more than usual & $11,596(41.5 \%)$ & $1.19(1.10-1.30)$ & $<0.001$ & $1.20(1.03-1.41)$ & 0.023 \\
\hline rather more than usual & $3,492(12.5 \%)$ & $1.43(1.27-1.60)$ & $<0.001$ & $1.80(1.46-2.22)$ & $<0.001$ \\
\hline much more than usual & $904(3.2 \%)$ & $1.96(1.62-2.36)$ & $<0.001$ & $2.44(1.76-3.39)$ & $<0.001$ \\
\hline \multicolumn{6}{|c|}{ Been losing confidence in self } \\
\hline not at all & $13,269(47.5 \%)$ & reference & $\mathrm{n} / \mathrm{a}$ & reference & $\mathrm{n} / \mathrm{a}$ \\
\hline no more than usual & $10,908(39.1 \%)$ & $1.12(1.04-1.22)$ & 0.003 & $1.09(0.94-1.26)$ & 0.272 \\
\hline rather more than usual & $2,986(10.7 \%)$ & $1.39(1.23-1.56)$ & $<0.001$ & $1.79(1.45-2.20)$ & $<0.001$ \\
\hline much more than usual & $765(2.7 \%)$ & $1.89(1.55-2.32)$ & $<0.001$ & $2.27(1.64-3.12)$ & $<0.001$ \\
\hline \multicolumn{6}{|c|}{ Been thinking of self as worthless } \\
\hline not at all & $19,055(68.3 \%)$ & reference & $\mathrm{n} / \mathrm{a}$ & reference & $\mathrm{n} / \mathrm{a}$ \\
\hline no more than usual & $6,793(24.3 \%)$ & $1.17(1.08-1.28)$ & $<0.001$ & $1.37(1.17-1.60)$ & $<0.001$ \\
\hline rather more than usual & $1,494(5.4 \%)$ & $1.44(1.24-1.66)$ & $<0.001$ & $1.94(1.50-2.52)$ & $<0.001$ \\
\hline much more than usual & $575(2.1 \%)$ & $1.81(1.42-2.29)$ & $<0.001$ & $1.74(1.18-2.58)$ & 0.006 \\
\hline \multicolumn{6}{|c|}{ Been feeling reasonably happy } \\
\hline more than usual & $2,662(9.5 \%)$ & reference & $\mathrm{n} / \mathrm{a}$ & reference & $\mathrm{n} / \mathrm{a}$ \\
\hline same as usual & $22,439(80.3 \%)$ & $1.10(0.97-1.26)$ & 0.141 & $1.13(0.89-1.45)$ & 0.311 \\
\hline less than usual & $2,283(8.2 \%)$ & $1.42(1.19-1.68)$ & $<0.001$ & $1.70(1.25-2.29)$ & 0.001 \\
\hline much less than usual & $550(2.0 \%)$ & $1.83(1.41-2.37)$ & $<0.001$ & $2.15(1.40-3.30)$ & $<0.001$ \\
\hline
\end{tabular}

Note: Adjusted for age, sex, ethnicity, body mass index, smoking status, high blood pressure, education level, alcohol intake, and exercise, and survey weighting.

In Table 4, by further exploring the variance between urban and rural areas, it was observed that more unexposed people lived in rural areas while more exposed people lived in urban environments. To be specific, the percentage of non-exposure in the city was $72.1 \%$ but $83.5 \%$ in the remote rural areas. The percentage of being exposed to passive smoking in one place was $22.2 \%$ in the city but $13.7 \%$ in the remote rural areas while the percentage of being exposed passive smoking in two places was $5.1 \%$ in the city but $2.4 \%$ in the remote rural areas. Interestingly, the percentage of being exposed to passive smoking in three places did not vary much across urban and rural settings. 
Table 4. Associations of urbanization levels and indoor passive smoking.

\begin{tabular}{lcccc}
\hline & Not Exposed & $\begin{array}{c}\text { Being Exposed } \\
\text { in 1 Place }\end{array}$ & $\begin{array}{c}\text { Being Exposed } \\
\text { in 2 Places }\end{array}$ & $\begin{array}{c}\text { Being Exposed } \\
\text { in 3 Places }\end{array}$ \\
\hline City $(>125,000$ people $)$ & $6,594(72.1 \%)$ & $2,029(22.2 \%)$ & $468(5.1 \%)$ & $53(0.6 \%)$ \\
Urban $(>10,000$ people) & $5,930(74.3 \%)$ & $1,643(20.6 \%)$ & $356(4.5 \%)$ & $51(0.6 \%)$ \\
Small accessible town & $1,861(76.5 \%)$ & $438(18.0 \%)$ & $120(4.9 \%)$ & $14(0.6 \%)$ \\
Small remote town & $1,343(77.6 \%)$ & $310(17.9 \%)$ & $64(3.7 \%)$ & $14(0.8 \%)$ \\
Rural & $2,882(81.7 \%)$ & $533(15.1 \%)$ & $104(3.0 \%)$ & $9(0.3 \%)$ \\
Remote rural & $2,658(83.5 \%)$ & $435(13.7 \%)$ & $75(2.4 \%)$ & $14(0.4 \%)$ \\
\hline
\end{tabular}

\subsection{Results on Never Smokers Only}

In Table 5, it was shown the relationships of indoor passive smoking and several self-reported CVDs in never smokers only. After full adjustment (see footnote in the table), it was observed that being exposed to passive smoking in more than two places could increase risks of angina (OR 2.43, 95\%CI 1.10-5.37, $p=0.028$ ), GHQ $\geq 12$ (OR 1.63, 95\%CI 1.15-2.30, $p=0.005$ ) and probably heart attack (OR 2.64, 95\%CI 0.99-7.00, $p=0.052)$ which indicated mostly in heart problems.

Table 5. Associations of indoor passive smoking and adult CVD in never smokers.

\begin{tabular}{lcccccc}
\hline & Yes & No & \multicolumn{2}{c}{ Being Exposed in 1 Place } & \multicolumn{2}{c}{ Being Exposed in $\geq 2$ Places } \\
\cline { 7 - 8 } & $\boldsymbol{n}(\boldsymbol{\%})$ & $\boldsymbol{n}(\mathbf{\%})$ & OR $(\mathbf{9 5 \% C I})$ & $\boldsymbol{p}$ value & OR (95\%CI) & $\boldsymbol{p}$ value \\
\hline Total CVD & $1,452(13.2 \%)$ & $9,452(86.8 \%)$ & $1.24(1.01-1.52)$ & 0.041 & $1.75(0.98-3.14)$ & 0.059 \\
Stroke & $258(2.4 \%)$ & $10,650(97.6 \%)$ & $1.38(0.89-2.16)$ & 0.151 & $1.53(0.41-5.66)$ & 0.523 \\
Angina & $442(4.1 \%)$ & $10,460(95.9 \%)$ & $1.21(0.86-1.71)$ & 0.270 & $2.43(1.10-5.37)$ & 0.028 \\
Heart attack & $255(2.3 \%)$ & $10,654(97.7 \%)$ & $1.37(0.88-2.14)$ & 0.167 & $2.64(0.99-7.00)$ & 0.052 \\
Heart murmur & $449(4.1 \%)$ & $10,459(95.9 \%)$ & $1.24(0.89-1.71)$ & 0.201 & $1.29(0.58-2.87)$ & 0.536 \\
Abnormal heart rhythms & $686(6.3 \%)$ & $10,220(93.7 \%)$ & $1.04(0.80-1.36)$ & 0.773 & $2.01(0.97-4.20)$ & 0.062 \\
Other heart problems & $244(2.2 \%)$ & $10,665(97.8 \%)$ & $1.67(1.14-2.45)$ & 0.009 & $0.37(0.05-2.66)$ & 0.326 \\
Diabetes & $579(5.3 \%)$ & $10,330(94.7 \%)$ & $1.12(0.85-1.48)$ & 0.422 & $0.93(0.45-1.91)$ & 0.838 \\
GHQ-12 scores $\geq 12$ & $3,244(32.7 \%)$ & $6,670(67.3 \%)$ & $1.12(0.97-1.29)$ & 0.110 & $1.63(1.15-2.30)$ & 0.005 \\
\hline
\end{tabular}

Note: Adjusted for age, sex, ethnicity, body mass index, high blood pressure, education level, alcohol intake, and exercise, and survey weighting.

To further explore events within mental health assessment scale (GHQ-12) in never smokers only, it was additionally observed that the indoor passive smoking risk effect by accounting the number of exposing places could also impact on sleep problems (more than usual: OR 2.53, 95\%CI 1.11-5.81, $p=0.028$ ), self-confidence (less than usual OR 2.76, 95\%CI 1.15-6.64, $p=0.023$ ), under strain constantly (more than usual: OR 2.87, 95\%CI 1.38-5.95, $p=0.005$ ), depressed (more than usual: OR 4.05, 95\%CI 1.75-9.37, $p=0.001$ ), and happiness (much less than usual: OR, 3.40, $95 \%$ CI $1.15-10.08, p=0.027$ ), in particular when being exposed in more than 2 places (for details see Table 6). 
Table 6. Associations of indoor passive smoking on adult mental health by GHQ-12 items in never smokers.

\begin{tabular}{|c|c|c|c|c|c|}
\hline \multirow{2}{*}{ GHQ-12 Items } & \multirow{2}{*}{$\begin{array}{c}\text { Yes } \\
n(\%)\end{array}$} & \multicolumn{2}{|c|}{ Being Exposed in 1 Place } & \multicolumn{2}{|c|}{ Being Exposed in $\geq 2$ Places } \\
\hline & & RRR $(95 \% C I)$ & $p$ value & RRR (95\%CI) & $p$ value \\
\hline \multicolumn{6}{|l|}{ Able to concentrate } \\
\hline better than usual & $779(2.8 \%)$ & reference & $\mathrm{n} / \mathrm{a}$ & reference & $\mathrm{n} / \mathrm{a}$ \\
\hline same as usual & $24,033(86.1 \%)$ & $1.13(0.74-1.72)$ & 0.575 & $1.84(0.33-10.24)$ & 0.489 \\
\hline less than usual & $2,658(9.5 \%)$ & $1.21(0.77-1.89)$ & 0.419 & $2.72(0.47-15.63)$ & 0.261 \\
\hline much less than usual & $450(1.6 \%)$ & $2.39(1.25-4.57)$ & 0.009 & $7.05(1.01-49.04)$ & 0.048 \\
\hline \multicolumn{6}{|l|}{ Lost sleep over worry } \\
\hline not at all & $10,249(36.7 \%)$ & reference & $\mathrm{n} / \mathrm{a}$ & reference & $\mathrm{n} / \mathrm{a}$ \\
\hline no more than usual & $13,310(47.6 \%)$ & $1.09(0.93-1.27)$ & 0.283 & $0.96(0.65-1.41)$ & 0.825 \\
\hline rather more than usual & $3,452(12.4 \%)$ & $1.37(1.11-1.70)$ & 0.003 & $1.28(0.72-2.26)$ & 0.401 \\
\hline much more than usual & $927(3.3 \%)$ & $1.51(1.04-2.19)$ & 0.031 & $2.53(1.11-5.81)$ & 0.028 \\
\hline \multicolumn{6}{|c|}{ Felt playing useful part in things } \\
\hline more than usual & $2,098(7.5 \%)$ & reference & $\mathrm{n} / \mathrm{a}$ & reference & $\mathrm{n} / \mathrm{a}$ \\
\hline same as usual & $22,872(82.1 \%)$ & $1.03(0.80-1.32)$ & 0.826 & $1.03(0.53-2.00)$ & 0.932 \\
\hline less than usual & $2,255(8.1 \%)$ & $1.27(0.92-1.77)$ & 0.150 & $1.59(0.68-3.70)$ & 0.284 \\
\hline much less than usual & $645(2.3 \%)$ & $1.77(1.06-2.95)$ & 0.029 & $2.42(0.68-8.55)$ & 0.171 \\
\hline \multicolumn{6}{|c|}{ Felt capable of making decisions } \\
\hline more than usual & $1,722(6.2 \%)$ & reference & $\mathrm{n} / \mathrm{a}$ & reference & $\mathrm{n} / \mathrm{a}$ \\
\hline same as usual & $24,202(86.6 \%)$ & $0.95(0.73-1.23)$ & 0.698 & $0.95(0.47-1.89)$ & 0.874 \\
\hline less than usual & $1,684(6.0 \%)$ & $1.21(0.84-1.73)$ & 0.301 & $1.49(0.61-3.62)$ & 0.379 \\
\hline much less than usual & $328(1.2 \%)$ & $1.35(0.68-2.68)$ & 0.395 & $0.62(0.08-5.04)$ & 0.655 \\
\hline \multicolumn{6}{|l|}{ Felt constantly under strain } \\
\hline not at all & $7,544(27.0 \%)$ & reference & $\mathrm{n} / \mathrm{a}$ & reference & $\mathrm{n} / \mathrm{a}$ \\
\hline no more than usual & $15,244(54.6 \%)$ & $0.99(0.84-1.18)$ & 0.943 & $1.10(0.67-1.80)$ & 0.705 \\
\hline rather more than usual & $4,182(15.0 \%)$ & $1.20(0.96-1.49)$ & 0.102 & $1.26(0.68-2.35)$ & 0.458 \\
\hline much more than usual & $958(3.4 \%)$ & $1.52(1.05-2.19)$ & 0.026 & $2.87(1.38-5.95)$ & 0.005 \\
\hline \multicolumn{6}{|c|}{ Felt couldn't overcome difficulties } \\
\hline not at all & $10,817(38.7 \%)$ & reference & $\mathrm{n} / \mathrm{a}$ & reference & $\mathrm{n} / \mathrm{a}$ \\
\hline no more than usual & $14,235(51.0 \%)$ & $1.05(0.91-1.22)$ & 0.505 & $0.99(0.67-1.45)$ & 0.944 \\
\hline rather more than usual & $2,257(8.1 \%)$ & $1.27(0.98-1.64)$ & 0.071 & $2.21(1.22-4.01)$ & 0.009 \\
\hline much more than usual & $611(2.2 \%)$ & $1.78(1.14-2.78)$ & 0.011 & $1.46(0.50-4.32)$ & 0.484 \\
\hline \multicolumn{6}{|c|}{ Able to enjoy day-to-day activities } \\
\hline more than usual & $1,403(5.0 \%)$ & reference & $\mathrm{n} / \mathrm{a}$ & reference & $\mathrm{n} / \mathrm{a}$ \\
\hline same as usual & $22,192(79.4 \%)$ & $0.96(0.71-1.30)$ & 0.781 & $1.74(0.66-4.56)$ & 0.261 \\
\hline less than usual & $3,478(12.5 \%)$ & $1.05(0.74-1.49)$ & 0.801 & $2.98(1.07-8.32)$ & 0.037 \\
\hline much less than usual & $863(3.1 \%)$ & $1.21(0.74-1.97)$ & 0.441 & $2.98(0.77-11.46)$ & 0.112 \\
\hline
\end{tabular}


Table 6. Cont.

\begin{tabular}{|c|c|c|c|c|c|}
\hline \multirow{2}{*}{ GHQ-12 Items } & \multirow{2}{*}{$\begin{array}{c}\text { Yes } \\
n(\%) \\
\end{array}$} & \multicolumn{2}{|c|}{ Being Exposed in 1 Place } & \multicolumn{2}{|c|}{ Being Exposed in $\geq 2$ Places } \\
\hline & & RRR $(95 \% C I)$ & $p$ value & RRR $(95 \% \mathrm{CI})$ & $p$ value \\
\hline \multicolumn{6}{|l|}{ Been able to face problems } \\
\hline more than usual & $1,212(4.3 \%)$ & reference & $\mathrm{n} / \mathrm{a}$ & reference & $\mathrm{n} / \mathrm{a}$ \\
\hline same as usual & $24,314(87.1 \%)$ & $1.06(0.75-1.49)$ & 0.754 & $1.03(0.45-2.33)$ & 0.951 \\
\hline less than usual & $1,975(7.1 \%)$ & $1.30(0.86-1.99)$ & 0.217 & $1.86(0.65-5.31)$ & 0.245 \\
\hline much less than usual & $420(1.5 \%)$ & $2.06(1.10-3.86)$ & 0.024 & $0.69(0.08-5.99)$ & 0.739 \\
\hline \multicolumn{6}{|c|}{ Been feeling unhappy and depressed } \\
\hline not at all & $11,937(42.7 \%)$ & reference & $\mathrm{n} / \mathrm{a}$ & reference & $\mathrm{n} / \mathrm{a}$ \\
\hline no more than usual & $11,596(41.5 \%)$ & $1.07(0.92-1.24)$ & 0.383 & $1.13(0.76-1.68)$ & 0.553 \\
\hline rather more than usual & $3,492(12.5 \%)$ & $1.23(0.99-1.52)$ & 0.059 & $2.44(1.53-3.91)$ & $<0.001$ \\
\hline much more than usual & $904(3.2 \%)$ & $1.72(1.17-2.53)$ & 0.006 & $4.05(1.75-9.37)$ & 0.001 \\
\hline \multicolumn{6}{|c|}{ Been losing confidence in self } \\
\hline not at all & $13,269(47.5 \%)$ & reference & $\mathrm{n} / \mathrm{a}$ & reference & $\mathrm{n} / \mathrm{a}$ \\
\hline no more than usual & $10,908(39.1 \%)$ & $0.96(0.83-1.11)$ & 0.584 & $1.21(0.82-1.77)$ & 0.336 \\
\hline rather more than usual & $2,986(10.7 \%)$ & $1.09(0.88-1.36)$ & 0.416 & $2.32(1.37-3.94)$ & 0.002 \\
\hline much more than usual & $765(2.7 \%)$ & $1.50(0.97-2.31)$ & 0.069 & $2.76(1.15-6.64)$ & 0.023 \\
\hline \multicolumn{6}{|c|}{ Been thinking of self as worthless } \\
\hline not at all & $19,055(68.3 \%)$ & reference & $\mathrm{n} / \mathrm{a}$ & reference & $\mathrm{n} / \mathrm{a}$ \\
\hline no more than usual & $6,793(24.3 \%)$ & $1.09(0.93-1.28)$ & 0.275 & $1.18(0.77-1.81)$ & 0.450 \\
\hline rather more than usual & $1,494(5.4 \%)$ & $1.16(0.87-1.55)$ & 0.326 & $1.99(1.01-3.92)$ & 0.047 \\
\hline much more than usual & $575(2.1 \%)$ & $1.50(0.91-2.46)$ & 0.113 & $1.52(0.55-4.20)$ & 0.421 \\
\hline \multicolumn{6}{|c|}{ Been feeling reasonably happy } \\
\hline more than usual & $2,662(9.5 \%)$ & reference & $\mathrm{n} / \mathrm{a}$ & reference & $\mathrm{n} / \mathrm{a}$ \\
\hline same as usual & $22,439(80.3 \%)$ & $1.03(0.82-1.30)$ & 0.816 & $1.16(0.62-2.17)$ & 0.638 \\
\hline less than usual & $2,283(8.2 \%)$ & $1.25(0.91-1.71)$ & 0.170 & $2.94(1.41-6.16)$ & 0.004 \\
\hline much less than usual & $550(2.0 \%)$ & $1.13(0.65-1.98)$ & 0.657 & $3.40(1.15-10.08)$ & 0.027 \\
\hline
\end{tabular}

Note: Adjusted for age, sex, ethnicity, body mass index, high blood pressure, education level, alcohol intake, and exercise, and survey weighting.

\subsection{Main Findings}

In the present study, recent evidence on cumulative effects of indoor passive smoking on CVD and psychological distress from a big Scottish Survey supported by the Scottish Government was provided. The cumulative effects were modeled by counting the number of indoor places. In this context, it was observed that being exposed to indoor passive smoking were significantly associated risks of stroke, angina, heart attack, abnormal heart rhythms, and psychological distress (GHQ $\geq 12$ ), in particular in more than two places of exposure. The significance remained for angina, psychological distress (GHQ $\geq 12$ ) and probably heart attack in never smokers. By exploring mental health specific indicators in detail, it was found that the cumulative risks impacted on sleep problems, 
self-recognition, making decisions, self-confidence, under strain constantly, depressed, happiness and self-worth. The significance remained for sleep problems, self-confidence, under strain constantly, depressed, and happiness in never smokers. In addition, people living in urban regions tended to be exposed at own home and other households than their counterparts living in the relative rural areas.

\subsection{Previous Research}

Since the 1980s, a few studies have shown the growing concern of passive smoking on Scottish health [10] including cardiorespiratory health [11,12], middle ear under pressure and effusion in children [13], coronary heart disease markers [14,15], lung function [16], and intermittent claudication in the middle-aged [17]. In addition to CVD such as stroke or coronary heart disease, less attention was paid to other forms of heart problems such as angina, heart attack, and abnormal heart rhythms in Scotland. However, previous research in the USA [18], Canada [19], and Greece [20] have indicated that clean indoor air environment could help reduce burden of angina and indoor passive smoking could impair symptomatic improvement in patients with chronic angina undergoing enhanced external counterpulsation [21]. Mostly passive smoking comes from sidestream smoke emitted from the burning tip of the cigarette and sidestream smoke is hazardous because it contains high concentrations of ammonia, benzene, nicotine, carbon monoxide, and many carcinogens [22]. Smoking could elicit both acute and chronic cardiac and vascular events due to the multiplicity of mechanisms involved hematological, neurohormonal, metabolic, hemodynamic, molecular genetic and biochemical pathways [23]. On the other hand, a few review articles have been supporting the view that smoking could act negatively on the heart causing atherosclerotic coronary alterations, focal myocardial lesions and arrhythmias [24,25]. Of note, these previous studies were mostly with rather small study sample.

\subsection{Strengths and Limitations}

The strength of this study lies in the very large study sample from across Scotland and could provide statistical power in modeling risk effects of indoor passive smoking. This is for the first time to examine the cumulative risk effect by counting the number of indoor places of exposure including own home, at work, and in other households. However, one limitation is that it was unable to assess the duration and the amount of being exposed to passive smoking from different indoor places including the historical records in the past during the childhood, adolescence, and/or early adulthood. Therefore, future research keeping the strengths and overcoming the limitations is recommended.

\section{Conclusions}

In summary, recent evidence has shown the cumulative risk effects of indoor passive smoking on $\mathrm{CV}$ and mental health in big Scottish population cohorts after the law banning smoking in public places since 2008. It has been known notoriously that being exposed to passive smoking is harmful for human health and a serious public health concern across the globe. Although the chance of exposure at work has been lessened due to the introduction of policy regulation, the risk effects from own home and other people's households seem to have persisted and affected human health including 
CVD (mostly heart problems) and mental health. Therefore, elimination of passive smoking from different indoor places should still be a top priority in future public health and perhaps housing policy. In particular, restriction of passive smoking in the households has proved to be protective for children in Scotland [3]. Wider implementation on restriction of indoor passive smoking to adults should therefore be further considered.

\section{Acknowledgments}

Ivy Shiue is supported by the Global Platform for Researchers Leaders scheme and would like to thank reviewers providing valuable feedback.

\section{Conflicts of Interest}

The author declares no conflict of interest.

\section{References}

1. Hamer, M.; Stamatakis, E.; Batty, G.D. Objectively assessed secondhand smoke exposure and mental health in adults: Cross-sectional and prospective evidence from the Scottish Health Survey. Arch. Gen. Psychiatr. 2010, 67, 850-855.

2. Sikorska-Jaroszyńska, M.H.; Mielnik-Błaszczak, M.; Krawczyk, D.; Nasiłowska-Barud, A.; Błaszczak, J. Passive smoking as an environmental health risk factor. Ann. Agric. Environ. Med. 2012, 19, 547-550.

3. Donnelly, P.; Whittle, P. After the smoke has cleared-Reflections on Scotland's tobacco control legislation. Public Health 2008, 122, 762-766.

4. Akhtar, P.C.; Haw, S.J.; Currie, D.B.; Zachary, R.; Currie, C.E. Smoking restrictions in the home and secondhand smoke exposure among primary schoolchildren before and after introduction of the Scottish smoke-free legislation. Tob. Control 2009, 18, 409-415.

5. The Scottish Government. Scottish Health Survey 1995; The Controller of HMSO and the Queen's Printer of Scotland: Scotland, UK, 1998.

6. The Scottish Health Survey. Available online: http:/www.scotland.gov.uk/Topics/Statistics/ Browse/Health/scottish-health-survey (accessed on 11 March 2014).

7. Scottish Health Survey: Design and Content. Available online: http://www.scotland.gov.uk/Topics/ Statistics/Browse/Health/scottish-health-survey/SurveyDesignContent (accessed on 11 March 2014).

8. Goldberg, D.P. Manual of the general health questionnaire. Nfer: London, UK, 1978.

9. The Scottish Health Survey 2011: Volume 3-Technical Report Available online: http://www. scotland.gov.uk/Publications/2012/09/8038/33 (accessed on 11 March 2014).

10. Tunstall-Pedoe, H.; Woodward, M.; Brown, C.A. The drinking, passive smoking, smoking deception and serum cotinine in the Scottish Heart Health Study. J. Clin. Epidemiol. 1991, 44, 1411-1414.

11. Hole, D.J.; Gillis, C.R.; Chopra, C.; Hawthorne, V.M. Passive smoking and cardiorespiratory health in a general population in the west of Scotland. BMJ 1989, 299, 423-427.

12. Chinn, S.; Rona, R.J. Quantifying health aspects of passive smoking in British children aged 5-11 years. J. Epidemiol. Community Health 1991, 45, 188-194. 
13. Strachan, D.P.; Jarvis, M.J.; Feyerabend, C. Passive smoking, salivary cotinine concentrations, and middle ear effusion in 7 year old children. BMJ 1989, 298, 1549-1552.

14. Tunstall-Pedoe, H.; Brown, C.A.; Woodward, M.; Tavendale, R. Passive smoking by self report and serum cotinine and the prevalence of respiratory and coronary heart disease in the Scottish heart health study. J. Epidemiol. Community Health 1995, 49, 139-143.

15. Pell, J.P.; Haw, S.; Cobbe, S.; Newby, D.E.; Pell, A.C.; Fischbacher, C.; Pringle, S.; Murdoch, D.; Dunn, F.; Oldroyd, K.; et al. Secondhand smoke exposure and survival following acute coronary syndrome: Prospective cohort study of 1261 consecutive admissions among never-smokers. Heart 2009, 95, 1415-1418.

16. Chen, R.; Tunstall-Pedoe, H.; Tavendale, R. Environmental tobacco smoke and lung function in employees who never smoked: The Scottish MONICA study. Occup. Environ. Med. 2001, 58, $563-568$.

17. Lippert, W.C.; Gustat, J. Clean Indoor Air Acts reduce the burden of adverse cardiovascular outcomes. Public Health 2012, 126, 279-285.

18. Naiman, A.; Glazier, R.H.; Moineddin, R. Association of anti-smoking legislation with rates of hospital admission for cardiovascular and respiratory conditions. CMAJ 2010, 182, 761-767.

19. Efstratiadis, S.; Kennard, E.D.; Kelsey, S.F.; Michaels, A.D. International EECP Patient Registry-2 Investigators. Passive tobacco exposure may impair symptomatic improvement in patients with chronic angina undergoing enhanced external counterpulsation. BMC Cardiovasc. Disord. 2008, 8 , doi:10.1186/1471-2261-8-23.

20. Pitsavos, C.; Panagiotakos, D.B.; Chrysohoou, C.; Skoumas, J.; Tzioumis, K.; Stefanadis, C.; Toutouzas, P. Association between exposure to environmental tobacco smoke and the development of acute coronary syndromes: The CARDIO2000 case-control study. Tob. Control 2002, 11, 220-225.

21. Lu, L.; Mackay, D.F.; Pell, J.P. Secondhand smoke exposure and intermittent claudication: A Scotland-wide study of 4,231 non-smokers. Heart 2013, 99, 1342-1345.

22. Eriksen, M.P.; LeMaistre, C.A.; Newell, G.R. Health hazards of passive smoking. Annu. Rev. Public Health 1988, 9, 47-70.

23. Taylor, B.V.; Oudit, G.Y.; Kalman, P.G.; Liu, P. Clinical and pathophysiological effects of active and passive smoking on the cardiovascular system. Can. J. Cardiol. 1998, 14, 1129-1139.

24. Leone, A. Cardiovascular damage from smoking: A fact or belief? Int. J. Cardiol. 1993, 38, 113-117.

25. Leone, A. Biochemical markers of cardiovascular damage from tobacco smoke. Curr. Pharm. Design 2005, 11, 2199-2208.

(C) 2014 by the authors; licensee MDPI, Basel, Switzerland. This article is an open access article distributed under the terms and conditions of the Creative Commons Attribution license (http://creativecommons.org/licenses/by/3.0/). 